\title{
The utility of diffusion tractography for speech preservation in laser ablation of the dominant insula: illustrative case
}

\author{
Timothy J. Kaufmann, MD, ${ }^{1}$ Vance T. Lehman, MD, ${ }^{1}$ Lily C. Wong-Kisiel, MD, ${ }^{2,3}$ Panagiotis Kerezoudis, $M D,{ }^{4}$ and Kai J. Miller, MD, $\mathrm{PhD}^{3-5}$ \\ Departments of ${ }^{1}$ Radiology, ${ }^{2}$ Neurology, ${ }^{3}$ Pediatric and Adolescent Medicine, ${ }^{4}$ Neurologic Surgery, and ${ }^{5}$ Biomedical Engineering, Mayo Clinic, Rochester, Minnesota
}

BACKGROUND Open surgical treatment of insular epilepsy holds particular risk of injury to middle cerebral artery branches, the operculum (through retraction), and adjacent language-related white matter tracts in the language-dominant hemisphere. Magnetic resonance imaging (MRI)-guided laser interstitial thermal therapy (LITT) is a surgical alternative that allows precise lesioning with potentially less operative risk. The authors presented the case of a 13-year-old girl with intractable, MRI-negative, left (dominant hemisphere) insular epilepsy that was treated with LITT. Diffusion tensor imaging (DTI) tractography was used to aid full posterior insular lesioning in the region of stereo electroencephalography-determined seizure onset while avoiding thermal injury to the language-related superior longitudinal fasciculus (SLF)/arcuate fasciculus (AF) and inferior fronto-occipital fasciculus (IFOF).

OBSERVATIONS DTI tractography was used successfully in planning insular LITT and facilitated a robust insular ablation with sharp margins at the interfaces with the SLF/AF and IFOF. These tracts were spared, and no neurological deficits were induced through LITT.

LESSONS Although it is technically demanding and has important limitations that must be understood, clinically available DTI tractography adds precision and confidence to insular laser ablation when used to protect important language-related white matter tracts.

https://thejns.org/doi/abs/10.3171/CASE21113

KEYWORDS laser interstitial thermal therapy; LITT; laser; insula; epilepsy; tractography; diffusion tensor imaging; DTI

Insular epilepsy can be difficult to identify because the insula connects extensively with other cortical structures, resulting in nonheterogeneous seizure presentations. ${ }^{1}$ The deep-seated location and extensive neural network can result in misleading scalp electroencephalography (EEG) seizure onset patterns. In the surgical evaluation for medically refractory insular epilepsy, stereo EEG (sEEG) is often preferred over grid-and-strip invasive monitoring to target this deep-seated structure and sample distant cortical surfaces to reveal accurate insular seizure onset. ${ }^{2}$ Insular epilepsy is often associated with cortical dysplasia but can also produce negative results on magnetic resonance imaging (MRI). ${ }^{2}$ Surgical options for insular epilepsy treatment include open resection and laser interstitial thermal therapy (LITT). Surgical hazards include the M2 branches of the middle cerebral artery and the nearby related superior longitudinal fasciculus/arcuate fasciculus (SLF/ AF) and inferior fronto-occipital fasciculus (IFOF), which are important language-related white matter tracts in the language-dominant cerebral hemisphere. LITT has some advantages over open surgery, including less risk to overlying middle cerebral artery branches and damage to overhanging frontal and temporal brain. However, the adjacent language SLF/AF and IFOF in the dominant cerebral hemisphere are vulnerable during open surgical intervention and LITT.

We present a case in which LITT was used to treat medicationrefractory insular epilepsy in the dominant (left) cerebral hemisphere. Diffusion tensor imaging (DTI) tractography was used to maximize ablation volume while explicitly protecting the SLF/AF and IFOF.

\section{Illustrative Case}

A 13-year-old, right-handed girl had onset of focal seizures at age 11. She was developmentally and cognitively intact and age appropriate. She was currently taking two antiepileptic medications and had previously tried four others.

ABBREVIATIONS AF = arcuate fasciculus; $C T=$ computed tomography; $D T I=$ diffusion tensor imaging; $E E G=$ electroencephalography; IFOF = inferior fronto-occipital fasciculus; LITT = laser interstitial thermal therapy; MRI = magnetic resonance imaging; sEEG = stereo EEG; SLF = superior longitudinal fasciculus.

INCLUDE WHEN CITING Published May 10, 2021; DOI: 10.3171/CASE21113.

SUBMITTED February 19, 2021. ACCEPTED February 22, 2021.

(C) 2021 The authors, CC BY-NC-ND 4.0 (http://creativecommons.org/licenses/by-nc-nd/4.0/). 


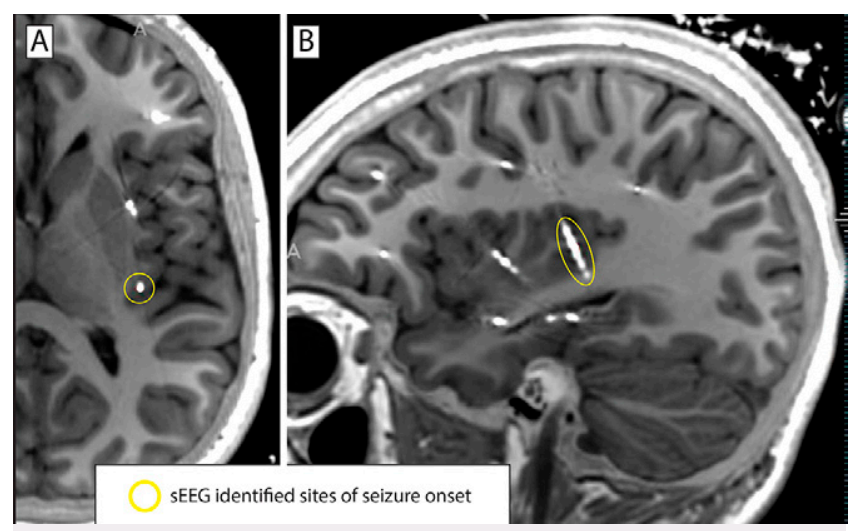

FIG. 1. The sEEG depth electrodes from prior CT are registered with preprocedural MRI in planning. The left posterior insular lead (yellow circles) registered seizure onset from five contact points, as shown in axial (A) and sagittal (B) images.

\section{Semiology}

Her seizures occurred most frequently while sleeping and started with a sucking or choking noise followed by eye deviation up and to the right. This was accompanied by right facial twitching and then bilateral eyelid fluttering and lip smacking. Seizures could secondarily generalize into a bilateral flexed tonic posture. Seizure frequency varied between daily occurrences and clusters of one to five occurrences every 2 to 5 weeks.

\section{Imaging}

Epilepsy protocol MRI did not reveal an epileptogenic lesion. There was no temporal lobe encephalocele on MRI or computed tomography (CT). ${ }^{18} \mathrm{~F}$-Fluorodeoxyglucose positron emission tomography depicted hypometabolism in the left temporal lobe.

\section{Electrophysiology}

Scalp EEG poorly localized seizure onset to the left frontotemporal region.

\section{Localization}

The choking semiology was suggestive of insula involvement. Because of the lack of an MRI-evident lesion, sEEG-implanted monitoring was performed with ten leads in the left limbic network and the insula. Three focal onset seizures were recorded from sEEG originating from the posterior insula (Fig. 1), with spread to the middle insula and then more broadly to the temporal neocortex and then mesial temporal regions.

\section{Surgical Planning}

After careful review of clinical and imaging findings at a multidisciplinary epilepsy board meeting, surgical intervention with LITT was planned to target the sEEG-identified posterior insular seizure onset zone. The patient received general anesthesia in an operating room equipped with an intraoperative MRI machine. A Leksell frame with fiducial box was placed, and CT venography was performed. The CT scan was registered to preoperative 3-T anatomical MRI (3T GE 750 W, GE Healthcare) and prior CT scans in which sEEG electrode leads were in place. The MRI included DTI with 30 directions and $2.5-\mathrm{mm}^{3}$ voxels. StealthViz with StealthDTI (Medtronic) was used to generate streamlines to represent the patient's left SLF/ $\mathrm{AF}$ and IFOF using deterministic tractography. Regions of interest were placed for the SLF/AF within the lateral aspect of the left cerebral hemispheric white matter over the anteroposteriorly oriented portion of the tract and over the superoinferiorly oriented portion of the tract in the posterosuperior temporal lobe/angular gyrus region. ${ }^{3} \mathrm{Re}$ gions of interest were placed for the IFOF in the anteroinferior frontal lobe and occipital lobe. ${ }^{3}$ The default StealthViz fractional anisotropy, tract length, and turning angle limits were used. The streamlines generated were registered to magnetization prepared rapid gradient echo anatomical imaging and a prior CT with sEEG leads and were used in laser applicator trajectory planning. Specifically, the trajectory direction and extent of planned ablation along this trajectory were modified to treat the seizure onset zone identified at SEEG (i.e., along the posterior insular lead) while preserving these two major language-related white matter tracts (Figs. 1 and 2).

\section{Laser Filament Implantation and Calibration}

A standard LITT procedure was performed. ${ }^{4,5}$ Using the Leksell arc for alignment, a drill hole was made in the skull, the dura was penetrated with cautery, and a Visualase (Medtronic) anchor bolt was placed in the skull. The Visualase laser cannula was placed to the planned depth, and the cannula was loaded with a 3-mm diffusing tip, 10-W maximum Visualase laser fiber. The patient was then placed in the intraoperative MRI scanner in a transmit-receive head coil, and T1-weighted inversion recovery gradient recalled echo imaging (brain volume imaging) was performed to verify laser cannula placement. Two-dimensional proton resonance frequency shift MR thermometry was set up in two planes and aligned with T2-weighted images along the long axis of the laser applicator, for a refresh time of 7 seconds. High $\left(90^{\circ} \mathrm{C}\right)$ temperature limits were placed on monitoring images with fiducial markers in the zones of highest expected heating to avoid tissue boiling, whereas low $\left(50^{\circ} \mathrm{C}\right)$ temperature limit fiducial markers were placed in a similar fashion to protect areas such as the left SLF/AF and IFOF from unwanted thermal injury.

\section{Ablation Protocol}

Test heating with the laser activated to $3.2 \mathrm{~W}$ was performed at each station before thermoablative heating. Ablation at four overlapping stations along the posterior insula was then performed through laser fiber drawbacks, with activation of the laser to $6 \mathrm{~W}$ for 1.5 to 3 minutes per station. We monitored tissue heating and expected cell damage zones in real time during ablations via Visualase software using the Arrhenius rate process model (Fig. 3). ${ }^{6}$ The expected SLF/AF and IFOF regions were protected from heating over $50^{\circ} \mathrm{C}$, although ablations were extended to the margins of both. The entire zone along the laser applicator at the posterior lobule of the insula reaching from the SLF/AF to the IFOF was ablated, including where the five sEEG contact points had previously detected seizure genesis. Postprocedural MRI depicted the ablation zone, an expected $1.1 \times 1.5 \times 2.2-\mathrm{cm}$ maximal diameter zone with thin peripheral water diffusion restriction and contrast enhancement.

\section{Postoperative Follow-Up}

There was no intracranial hemorrhage or middle cerebral artery territory infarction. The patient was awakened from anesthesia and there was no complication. The patient had no language or 

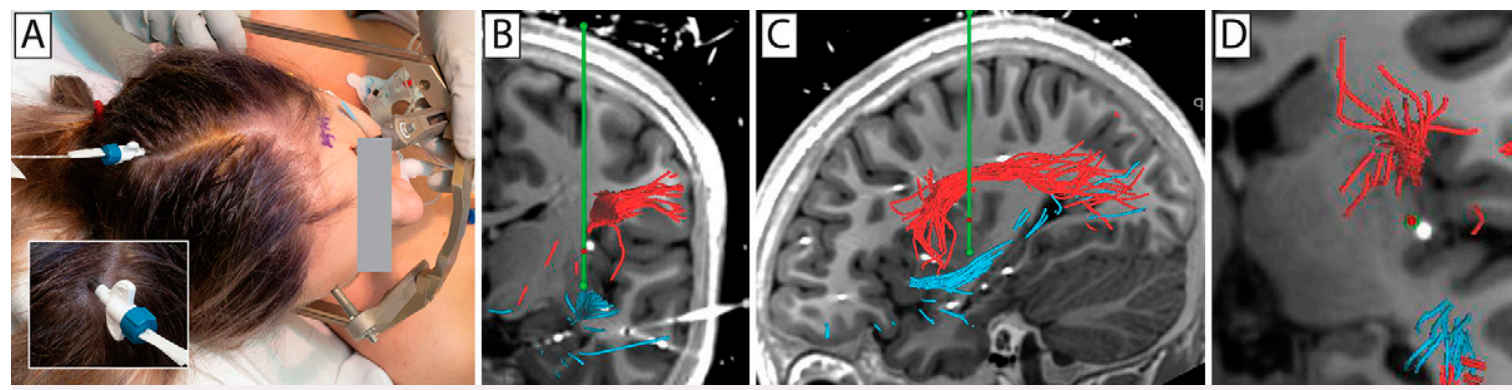

FIG. 2. A: Intraoperative photograph of the skull anchor bolt and laser applicator through it. B-D: MRI fused with sEEG CT, with DTI tracts overlaid for use in surgical planning. The green line depicts the planned laser applicator trajectory to the left posterior insula in coronal (B) and sagittal (C) planes and passes through the green circle (D). The SLF/AF is represented in red, and the IFOF is represented in blue.

sensorimotor deficit postoperatively. She was discharged home on postoperative day 1 and instructed to return for a 3-month followup visit.

\section{Discussion}

Epilepsy surgery or ablation in the dominant cerebral hemisphere poses significant risk of injury to cortical and white matter areas that serve language function. Although our understanding of human language function as it maps to the brain continues to grow and evolve, ${ }^{7-10}$ it has long been understood that surgical injury to Broca's area in the dominant inferior frontal gyrus, Wernicke's area centered in the dominant posterosuperior temporal lobe, and white matter tracts that convey language-related information between or near these two areas can cause devastating and permanent deficits. Such language-related white matter tracts include the SLF, which has been separated into four subcomponents (SLF I-III and the AF), ${ }^{8,11}$ and the IFOF. The SLF III and AF play a major role in the dorsal language stream, and the IFOF is the major tract of the ventral language stream. ${ }^{8,10}$

Although other cortical and subcortical areas are involved in language function, these two particular tracts are known to be of primary importance and should be protected during surgery or ablation. DTI tractography was used in our patient to visualize these two major tracts in preoperative planning and allow us to fully treat the left, language-dominant hemisphere and insular seizure onset zone while avoiding thermal damage to these tracts with spatial precision. This procedure provided a less invasive means of epilepsy surgery in an area in which it can be dangerous to operate because of overlying M2 middle cerebral artery branches, the necessity to dissect the Sylvian fissure while retracting the overlying cortex, and the presence of language-related white matter tracts.

There have been a few published cases of insular thermal ablations for patients with epilepsy., ${ }^{2,13}$ An advantage of insular LITT over open surgery may be less risk to middle cerebral artery branches. Although thermal injury to arterial walls while using LITT would be catastrophic, the circulation of arterial blood flow actively cools the vessel and makes this occurrence less likely. ${ }^{4}$ Perioperative morbidity and length of hospital stay after making a small drill hole are, on average, much less than after open craniotomy. LITT can be performed with controlled precision using MRI guidance, and the relative absence of brain shift (sagging) after a drill hole (compared with an open craniotomy) preserves neuronavigational and image-guided accuracy.

LITT, which creates thermal tissue injury through the absorption and conversion of light energy to heat energy within brain parenchyma, has a relatively sharp margin between ablated and nonablated tissues. It is most well suited for small lesions that do not exceed the diameter of the ablation zone created by the laser, which varies by laser vendor and wattage used but is on the order of 1.5 to $2 \mathrm{~cm}$. However, larger or more complex-shaped lesions may be addressed by placing more than one laser cannula. LITT with MR thermometry has been used for several years to treat epilepsy in children and adults, primarily for lesional epilepsy (e.g., mesial temporal sclerosis) but also for sEEG-localized seizure onset zones with MRI-negative presentations. ${ }^{5,14-16}$ Several previous cases of LITT used for insular epilepsy have been reported, both lesional and nonlesional., ${ }^{2,12}$ However, our use of DTI tractography to aid insular LITT in the dominant hemisphere is the first such case reported (to our knowledge).

\section{Observations}

Although no underlying epileptogenic MRI-evident lesion was discernible in our patient, a focal seizure onset zone was identified with sEEG and could be accurately targeted using the multimodal image registrations described and illustrated previously (Figs. 1 and 2). It was an ideal ablation target for LITT except for its proximity to languagerelated white matter tracts in the dominant cerebral hemisphere in this cognitively normal child. Whereas a transopercular (lateral-to-medial) trajectory with LITT may have put these language tracts at less risk, this approach allows a limited volume of insular ablation for each cannula placed. Furthermore, such an approach traverses the Sylvian fissure, carrying an increased risk of vessel injury during cannula placement and increasing the likelihood of inadvertent ablation of deeper (more medial) structures. Therefore, we chose the oblique trajectory illustrated in Figs. 2 and 3. DTI tractography of the language-related tracts then enabled precise ablation up to, but not involving, them. Postprocedural MRI (Fig. 3D) confirmed ablation throughout the epileptogenic zone but not encroaching upon regions of the SLF/AF or IFOF. DTI tractography increased our confidence in our ablation plan and helped to keep the patient safe. Safety shutdown cursors placed on the regions of the important white matter tracts, which had been identified by tractography, further contributed to patient safety (Fig. 3A). 

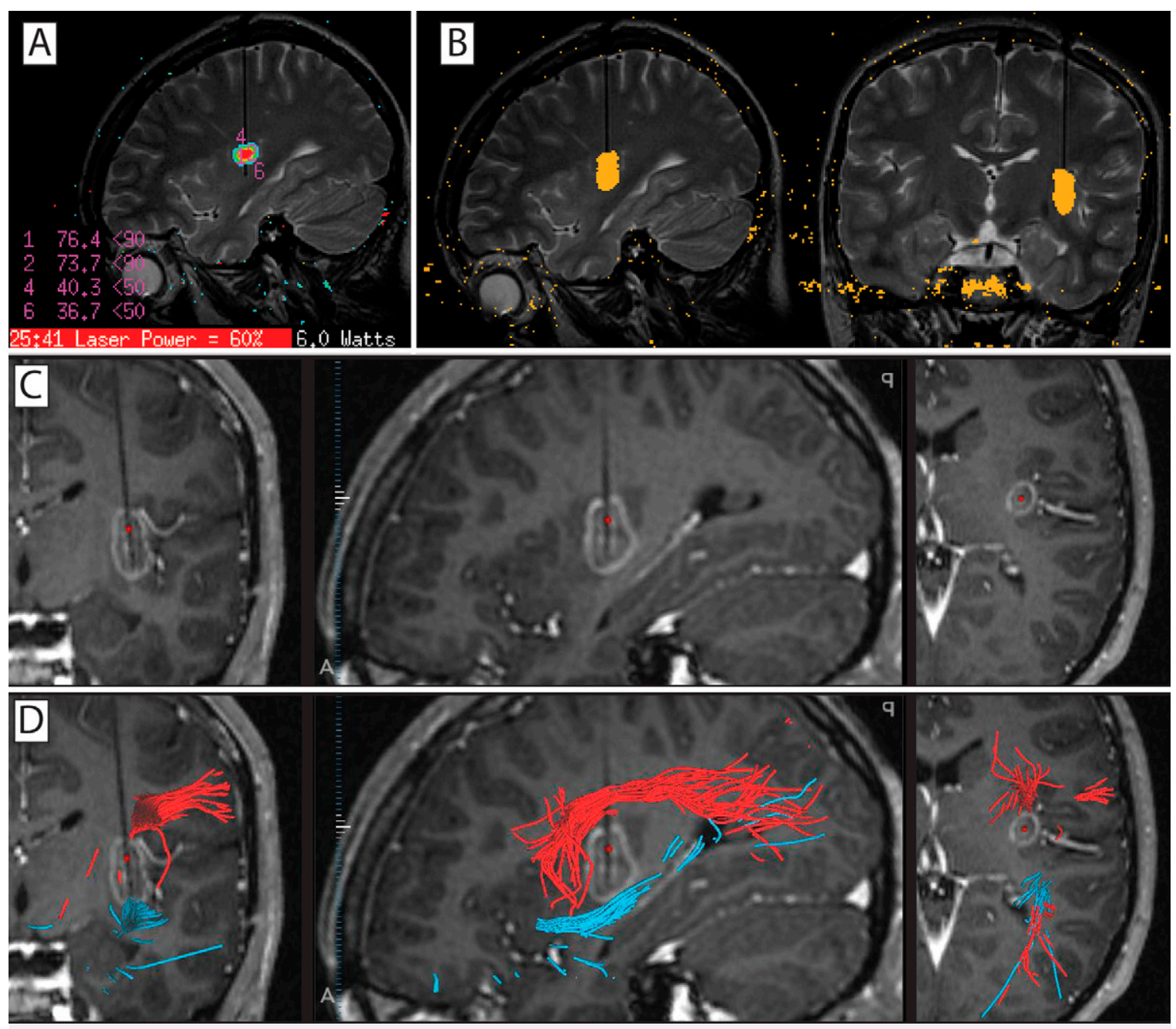

FIG. 3. A: MR thermometry data are superimposed on a T2-weighted image showing local tissue heating in the left insula around this 3-mm diffusing tip laser fiber during active ablation (red area shows hottest temperature). Safety shutdown cursor locations are shown by overlaid purple numbers. B: Estimated permanent cell damage is also updated in real time during ablation (represented in gold) and superimposed on these oblique sagittal and coronal T2-weighted images during ablation. C: Three-plane post-gadolinium T1-weighted imaging with the laser applicator still in place showing the ablated zone with expected thin peripheral enhancement, which extends from superior to inferior insula. D: Registered with the postoperative images (C), the SLF/AF (red) and IFOF (blue) from preoperative imaging show the ablation zone extending from the SLF to the IFOF, sparing both white matter tracts.

\section{Lessons}

Eloquent cortical areas of the brain may be identified by intraoperative cortical mapping or by functional MRI. Just as important to critical human functioning are the underlying white matter tracts that connect these cortical areas. DTI tractography, if used carefully and with an understanding of its limitations, can increase confidence in surgical plans and help protect critical functions. It was used in this case to enable a full posterior insular ablation up to, but not into, the adjacent SLF/AF and IFOF tracts.

Caution must be used when DTI tractography is used in surgical planning, however. What is generated by image postprocessing software are streamlines, which are continuous lines of high fractional anisotropy (suggesting intact white matter) and maps from one user-selected point to another in the brain. These streamlines approximate true anatomical white matter tracts to one degree but involve many simplifications and limitations in the process of their generation, leading to the common occurrence of false-positive (spurious) and false-negative (not seen but present) tract representations. The user must optimize outputted tracts and compare results with expected anatomy to the degree possible, first using only high-quality, non-motion-compromised DTI data and quality software. Surgical interpretation of DTI tracts should agree with the known location of critical pathways, serving to refine general expected anatomy to the specific nuance of the individual patient.

Technical aspects of DTI tractography, including deterministic versus probabilistic techniques, are beyond the scope of this report but are widely available elsewhere.,17-19 In brief, DTI for tractography is technically demanding, and we use only our optimal 3-T clinical scanners for surgical planning purposes. DTI has 
relatively low spatial resolution relative to standard anatomical $\mathrm{MRI}$, which is a challenge to tractography when the relatively large voxels may include fibers from multiple tracts, not just the one of interest. ${ }^{17}$ However, it is ideal to use isotropic voxels in DTI used for tractography, even if it means diminished in-plane spatial resolution. ${ }^{17}$ Thus, any increase in scanner gradient strength and signal one can obtain will be helpful, and the advent of head-only compact scanners may be particularly useful. ${ }^{20}$ Probabilistic tractography software has some advantages over deterministic tractography, although it has its own caveats. ${ }^{17}$ At the time of this writing, and to our knowledge, it is not available in a US Food and Drug Administration-approved software package. This case demonstrates that deterministic tractography with widely available off-the-shelf software using default settings is feasible for LITT planning in the insular region. Finally, white matter streamlines or tracts must be generated from tractography software that is compatible with the available neuronavigational software for a given practice.

Performing DTI tractography and including it in a surgical plan can add precision and confidence to ablation performed by MRIguided LITT. It is particularly valuable in potentially dangerous areas, such as the language-dominant insula.

\section{Acknowledgments}

Dr. Miller is supported by NIH-NCATS Clinical and Translational Science Award KL2 TR002379.

\section{References}

1. Jobst BC, Gonzalez-Martinez J, Isnard J, et al. The insula and its epilepsies. Epilepsy Curr. 2019;19(1):11-21.

2. Hale AT, Sen S, Haider AS, et al. Open resection versus laser interstitial thermal therapy for the treatment of pediatric insular epilepsy. Neurosurgery. 2019;85(4):E730-E736.

3. Hijaz TA, McComb EN, Badhe S, et al. Diffusion tensor imaging tractography tutorial and introduction to major white matter tract anatomy and function. Neurographics. 2019;9(1):62-74.

4. Medina LS, Alvarado E, Pacheco E, et al. Minimally invasive MR imaging-guided stereotactic laser thermal ablation in neurosurgical cases of pediatric lesional epilepsy: a didactic step-by-step practical approach. Neurographics. 2016;6(6):390-399.

5. North RY, Raskin JS, Curry DJ. MRI-guided laser interstitial thermal therapy for epilepsy. Neurosurg Clin N Am. 2017;28(4):545-557.

6. Sherar MD, Moriarty JA, Kolios MC, et al. Comparison of thermal damage calculated using magnetic resonance thermometry, with magnetic resonance imaging post-treatment and histology, after interstitial microwave thermal therapy of rabbit brain. Phys Med Biol. 2000;45(12):3563-3576.

7. Hickok G, Poeppel D. Dorsal and ventral streams: a framework for understanding aspects of the functional anatomy of language. Cognition. 2004;92(1-2):67-99.

8. Dick AS, Bernal B, Tremblay P. The language connectome: new pathways, new concepts. Neuroscientist. 2014;20(5):453-467.

9. Saur D, Kreher BW, Schnell S, et al. Ventral and dorsal pathways for language. Proc Natl Acad Sci U S A. 2008;105(46):18035-18040.
10. Hill VB, Cankurtaran CZ, Liu BP, et al. A practical review of functional MRI anatomy of the language and motor systems. AJNR Am J Neuroradiol. 2019;40(7):1084-1090.

11. Makris N, Kennedy DN, Mclnerney S, et al. Segmentation of subcomponents within the superior longitudinal fascicle in humans: a quantitative, in vivo, DT-MRI study. Cereb Cortex. 2005;15(6):854-869.

12. Perry MS, Donahue DJ, Malik SI, et al. Magnetic resonance imagingguided laser interstitial thermal therapy as treatment for intractable insular epilepsy in children. J Neurosurg Pediatr. 2017;20(6):575-582.

13. Alexander H, Cobourn K, Fayed I, Oluigbo CO. Magnetic resonance-guided laser interstitial thermal therapy for the treatment of nonlesional insular epilepsy in pediatric patients: technical considerations. Pediatr Neurosurg. 2020;55(3):155-162.

14. Hoppe C, Witt JA, Helmstaedter C, et al. Laser interstitial thermotherapy (LiTT) in epilepsy surgery. Seizure. 2017;48:45-52.

15. Remick M, McDowell MM, Gupta K, et al. Emerging indications for stereotactic laser interstitial thermal therapy in pediatric neurosurgery. Int J Hyperthermia. 2020;37(2):84-93.

16. Tao JX, Wu S, Lacy M, et al. Stereotactic EEG-guided laser interstitial thermal therapy for mesial temporal lobe epilepsy. J Neurol Neurosurg Psychiatry. 2018;89(5):542-548.

17. Mukherjee P, Chung SW, Berman JI, et al. Diffusion tensor MR imaging and fiber tractography: technical considerations. AJNR Am J Neuroradiol. 2008;29(5):843-852.

18. Mukherjee P, Berman JI, Chung SW, et al. Diffusion tensor MR imaging and fiber tractography: theoretic underpinnings. AJNR Am J Neuroradiol. 2008;29(4):632-641.

19. Essayed WI, Zhang F, Unadkat $P$, et al. White matter tractography for neurosurgical planning: a topography-based review of the current state of the art. Neuroimage Clin. 2017;15:659-672.

20. Foo TKF, Laskaris E, Vermilyea M, et al. Lightweight, compact, and high-performance 3T MR system for imaging the brain and extremities. Magn Reson Med. 2018;80(5):2232-2245.

\section{Disclosures}

The contents of this study are solely the responsibility of the authors and do not necessarily represent the official views of the National Institutes of Health. The authors report no conflict of interest concerning the materials or methods used in this study or the findings specified in this paper.

\section{Author Contributions}

Conception and design: Kaufmann, Lehman, Kerezoudis, Miller. Acquisition of data: all authors. Analysis and interpretation of data: Kaufmann, Lehman, Kerezoudis. Drafting the article: Kaufmann, WongKisiel, Kerezoudis. Critically revising the article: all authors. Reviewed submitted version of manuscript: all authors. Approved the final version of the manuscript on behalf of all authors: Kaufmann. Study supervision: Kaufmann.

\section{Correspondence}

Timothy J. Kaufmann: Mayo Clinic, Rochester, MN. kaufmann.timothy@ mayo.edu. 